\title{
Time-of-flight Near-infrared Spectroscopy for Nondestructive Measurement of Internal Quality in Grapefruit
}

\author{
Yohei Kurata \\ College of Bioresource Sciences, Nihon University, Fujisawa, Kanagawa 252-0880, Japan \\ Tomoe Tsuchida and Satoru Tsuchikawa ${ }^{1}$ \\ Graduate School of Bioagricultural Sciences, Nagoya University, Nagoya, Aichi 464-8601, Japan
}

\begin{abstract}
AdDitional INDEX wORDs. optical characteristics, cross correlation function, partial least squares regression
Abstract. We proposed a technique combining time-of-flight (TOF) and near-infrared spectroscopy (NIRS), termed TOF-NIRS, capable of measuring the time-resolved profiles of near-infrared (NIR) light with nanosecond resolution. Analysis of the variation in time-resolved profiles was used to estimate soluble solids concentration (SSC) and acidity in grapefruit (Citrus paradisi), and the prediction accuracy was compared with the conventional NIR measurement device. In data processing, the cross-correlation function, which evaluated the similarity between the reference and transmitted beams, was introduced as an explanatory variable for partial least squares regression. TOF-NIRS predicted both SSC and acidity in grapefruit with higher precision than the conventional NIR measurement with respective $r$ values of 0.72 and 0.85 . Specifically, the superiority of TOF-NIRS was attributed to measurement time and prediction accuracy in determining acidity.
\end{abstract}

A time-resolved measurement, or time-domain system, could provide the TOF information of detected electromagnetic beams. The TOF photon technique enables determination of certain details of absorption and scattering phenomena in various samples. Many applications of time-resolved reflectance and transmittance have been widely investigated for use in biological tissues or agricultural products (Valero et al., 2004; Vanoli et al., 2009). Leonardi and Burns (1997, 1999a, 1999b) performed quantitative measurements in scattering media on the basis of TOF analysis and described the analytical possibilities of the technique. Nicolai et al. (2008) reported the soluble solids content and firmness of pear (Pyrus communis cv. Conference) by using a time-resolved technique, in which fruit or agricultural products were considered to be the scattering media. Furthermore, it was reported that the time-resolved profile was very efficient for estimating the absorption and scattering coefficients (Gributs and Burns, 2003a, 2003b; Pandozzi and Burns, 2007).

Recently, we proposed a technique combining TOF and NIRS, termed TOF-NIRS, capable of measuring the timeresolved profiles of NIR light with nanosecond resolution. The behavior of a NIR-pulsed laser beam into various fruit was compared with assess the absorption/scattering characteristics of NIR radiation (Kurata et al., 2008). Furthermore, the absorption/ scattering conditions of NIR radiation in grapefruit were analyzed in detail using the cross-correlation function, which is used to calculate the similarity between reference and transmitted light (Kurata and Tsuchikawa, 2009).

In previous research, Tsuchikawa and Hamada (2004) applied TOF-NIRS for the detection of sugar and acidity in apple

Received for publication 18 Sept. 2012. Accepted for publication 22 Mar. 2013. This research was partially supported by the Ministry of Education, Science, Sports and Culture, Grant-in-Aid for Scientific Research (No. 22248020 to S.T.) and (No. 23880027 to Y.K.).

${ }^{1}$ Corresponding author. E-mail: st3842@agr.nagoya-u.ac.jp.
(Malus sylvestris var. domestica), in which attenuance of peak maxima, time delay of peak maxima, and variation of full width at half maximum of the time-resolved profile were used as explanatory variables for multiple linear regression, principle component regression, and partial least squares regression (PLSR) analysis. It was possible to predict both the sugar and acid contents in apple with high precision using TOF-NIRS.

In our previous paper (Kurata and Tsuchikawa, 2009), a Q-switched neodymium yttrium aluminum garnet (Nd:YAG) laser with high-output energy was used as the laser source. The principle of Q-switched Nd:YAG laser is briefly described below. The optical pumping continues as the light energy in the laser resonator is attenuated. During optical pumping, the number of atoms in an excited state in the laser medium increases. As a result, high-intensity output is obtained from the Q-switched $\mathrm{Nd}$ :YAG laser by raising the $\mathrm{Q}$ factor of the resonator. Here, the laser output of a time-resolved profile was composed of certain peaks because of the excitation characteristics of the Nd:YAG laser. To compensate for this fluctuation, the smoothed timeresolved profile was averaged over a specified time period. The multiple smoothing calculations were done not only for a singleshot pulse, but also for a period of several seconds. Such measurement is unsuitable for online measurement, although the pulse laser itself only requires nanosecond resolution. The timeresolved profile of a single-shot pulse is useful in investigating phenomena in a short time domain.

In this report, a diode-pumped solid-state laser with highoutput energy and single-pulse operation was used as the laser source. Analysis of the variation in the single-pulsed laser in a short time domain was used to estimate SSC and acidity in grapefruit, in which the wavelength was varied to obtain the best prediction model. With respect to validation of the PLSR model, TOF-NIRS was compared with the conventional NIR procedure in interactance mode. These basic comparative measurements are essential for constructing a precise, nondestructive online measurement for fruit. 


\section{Materials and Methods}

OUtLINe OF TOF-NIRS DEviCE. Figure 1 shows the TOF-NIRS measurement system. A diode-pumped solid state laser (Wedge HB-532-SC; Bright Solutions, Cura Carpignano, Italy) was used as the light source under the following conditions: output energy of $1.5 \mathrm{~mJ}$ per pulse at $532 \mathrm{~nm}$, a pulse width of $1.5 \mathrm{~ns}$ at half height, and a pulse repetition frequency of $1 \mathrm{kHz}$. The laser output wavelength was tuned from 750 to $860 \mathrm{~nm}$ (every $10 \mathrm{~nm}$ ) using dye-doped plastic (LDS798 etc.; Seiko Electric Co., Fukuoka, Japan), as described in detail by Oki et al. (2004).

Intensities of the transmitted beam through the sample and the reference beam were measured using two avalanche photodiode detectors (C5658; Hamamatsu Photonics, Shizuoka, Japan). The reference beam generated a trigger signal, and specific neutral density filters were used to avoid overloading the detector. One of the avalanche photodiode detectors was directly in contact with the grapefruit sample. The light beam was passed in the center of the grapefruit and the transmitted output was detected at the center of the opposite face. The intensity of the reference and transmitted beams was recorded up to $50 \mathrm{~ns}$.

OUTLINE OF THE TIME-RESOLVED PROFILE AND THE CROSS-CORRELATION FUNCTION. "Time-resolved profile" refers to the variation in intensity of the transmitted radiation of the pulsed laser beam with time. Figure 2A shows the time-resolved profiles of a grapefruit and reference beam. The laser output varies for each measurement; therefore, the transmitted radiation is not directly comparable between measurements. Thus, we assessed the similarity between the reference beam and transmitted beam so as to account for the variability in laser output. Figure $2 \mathrm{~B}$ shows the cross-correlation function between the reference beam and the transmitted beam using grapefruit. Here, the cross-correlation function, $R_{x y}(\tau)$, of the continuous functions $x(\tau)$ and $y(\tau)$, which correspond to the timeresolved profile for the reference beam and the transmitted beam from the grapefruit, respectively, is defined as follows:

$$
R_{x y}(\tau)=\lim _{T \rightarrow \infty} \int_{0}^{T} x(t) y(t+\tau) d t
$$

where $\tau$ is the time delay between the continuous functions $x(t)$ and $y(t)$.

OUTLINE OF THE CONVENTIONAL NIR SPECTROPHOTOMETER. Figure 3A shows the conventional NIR device in interactance mode (Fruits Selector K-BA 100R; Kubota Co., Osaka, Japan), which covers the visible and NIR ranges from 500 to $1010 \mathrm{~nm}$ (2-nm increments). Samples were irradiated from the side by

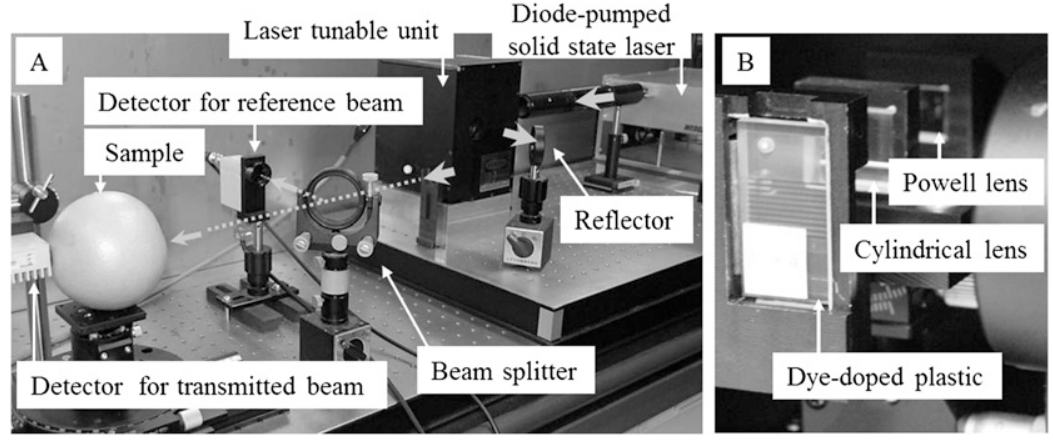

Fig. 1. (A) Outline of time-of-flight near-infrared measurement system. The time-resolved profile from 750 to $860 \mathrm{~nm}$ (every $10 \mathrm{~nm}$ ) was obtained by changing the wavelength in the laser tunable unit (B) that included the dye-doped plastic.
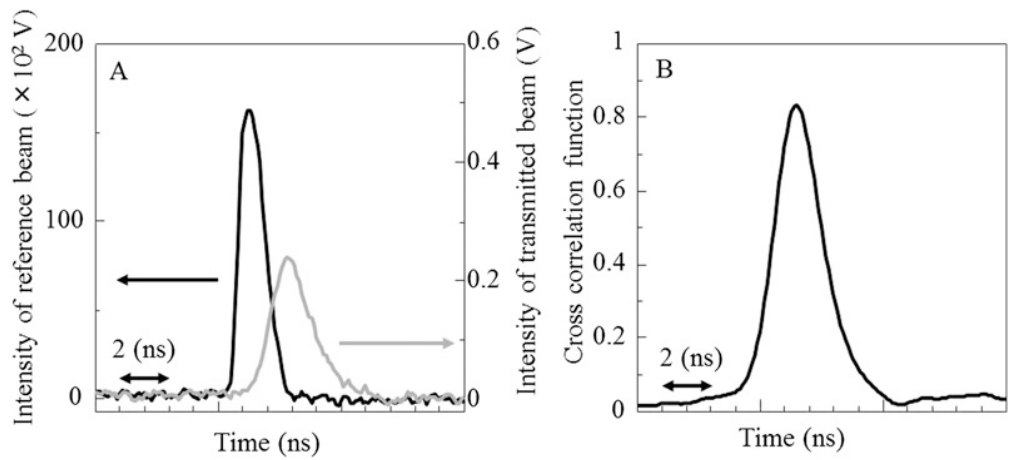

Fig. 2. (A) Time-resolved profile of transmitted beam from grapefruit (gray line) and reference beam (black line) at a wavelength of $790 \mathrm{~nm}$. (B) The results for calculating the cross-correlation function between the reference beam and transmitted beam.
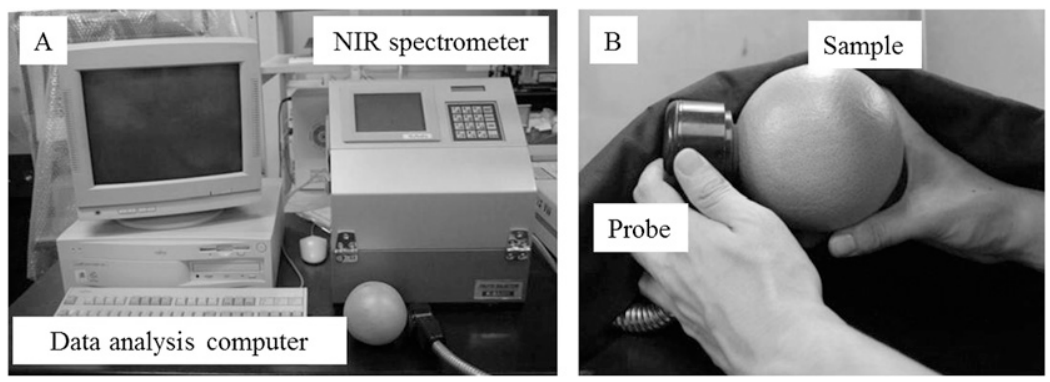

Fig. 3. (A) Outline of the conventional near-infrared (NIR) device. (B) The situation of spectra acquisition from grapefruit and the spectra from 500 to $1010 \mathrm{~nm}$ (2-nm increments) were obtained by this measurement device. the light source (Fig. 3B). The interactance probe was in direct contact with the samples. The optical fiber was fixed onto the experimental table for noise reduction. Because grapefruit are large, with a thick peel, sufficient incident light (that is, sufficient exposure time) was required to obtain valid spectra. Measurements required 20 to $30 \mathrm{~s}$ per sample.

GrAPEFRUIT SAMPLE. A total of 70 commercial white grapefruit, harvested in Florida, were used in this study. The fruit samples were stored at $20^{\circ} \mathrm{C}$ for $2 \mathrm{~d}$ to ensure a constant internal temperature. In this experiment, we used grapefruit of roughly the same diameter (average $92.1 \mathrm{~cm}$ ) to avoid the effect of sample size on the spectral data. After the TOF-NIRS and conventional NIR measurements, a portion of the flesh illuminated 
during measurement was squeezed to obtain the juice. The juice was obtained from both sides of the illuminated quarter and pooled. The SSC value of the juice was measured using a digital refractometer (Model PR-320; ATAGO, Tokyo, Japan). Acidity was measured using sodium hydroxide titration. These measurements were performed in triplicate and reported as average values. Table 1 shows the descriptive statistics for the SSC and acidity determinations.

Data ANALYSIS. In the TOF-NIRS measurement, the crosscorrelation function was used in data analysis as an explanatory variable. In conventional NIR spectrophotometry measurement, visible-NIR spectra were used in the data analysis. PLSR analysis was performed as a multivariate analysis. Before statistical analysis, we assessed the relationship between grapefruit diameter and the measured parameters (SSC and acidity). No significant correlation between SSC or acidity and diameter was observed. However, a significant, but weak, correlation between SSC and acidity $(r=0.43)$ was observed. Unscrambler software (Version 9.6; CAMO, Oslo, Norway) was applied for spectral pretreatment and quantitative analysis with PLSR. In the PLSR analysis, various preprocessing methods were applied for baseline offset, noise reduction, and smoothing. We investigated the optimal parameters for predicting SSC and acidity using TOF-NIRS. Although transformation of the explanatory variable (for example, first derivative, secondary derivative, etc.) was also important to determine a good calibration equation, we paid specific attention to the original spectral curve of the cross-correlation function, because it is difficult to find spectroscopic meaning in the transformed data. With respect to PLSR analysis of the conventional NIR spectrophotometry data, smoothing, multiplicative scatter correction, and second derivative were performed to obtain the best preprocessing results.

\section{Results and Discussion}

Evaluation OF SSC AND ACIDITY USING TOF-NIRS. Table 2 shows the PLSR analysis results for predicting SSC and acidity using TOF-NIRS and the conventional NIR technique. For

Table 1. The statistical data of soluble solids concentration (SSC) and acidity in samples of 70 commercial white grapefruit harvested in Florida.

\begin{tabular}{lcccc}
\hline Parameter & Avg & Maximum & Minimum & SD \\
\hline SSC (\%) & 9.69 & 11.20 & 8.50 & 0.51 \\
Acidity (\%) & 1.35 & 1.72 & 0.95 & 0.14 \\
\hline
\end{tabular}

TOF-NIRS, the best result was obtained at $\lambda=770 \mathrm{~nm}$, in which the coefficient of determination $(r)$ between measured and predicted SSC and the root mean square error of cross-validation (RMSECV) were $0.72 \%$ and $0.35 \%$, respectively. With respect to the acidity result at $\lambda=760 \mathrm{~nm}, r$ and RMSECV were $0.85 \%$ and $0.06 \%$, respectively. RMSECV of both chemical parameters in grapefruit using the TOF-NIRS prediction model was superior to that of the NIR spectrophotometry prediction model. In the conventional NIR, the reflected light that provided the analytical data was not fully detected because of the peel thickness, whereas with TOF-NIRS, the transmitted light could be detected. The ratio of performance to standard deviation (RPD) of SSC and acidity using TOF-NIRS was 1.49 and 2.19, respectively. An RPD greater than 1.5 should be attainable for an initial screening tool; therefore, TOF-NIRS might be acceptable for practical use such as for an online measurement system. Because the wavelength of the incident pulse depends on the optical characteristic of the dye-doped plastic, the pulsed laser radiation does not correspond to specific absorption bands resulting from the chemical constituents of grapefruit. Therefore, the theoretical background for the prediction of SSC and acidity with the mentioned wavelengths might be the result of light refraction or scattering in samples.

We should also consider that absorption resulting from $\mathrm{OH}$ groups appears from 750 to $800 \mathrm{~nm}$, which might relate to the prediction of the internal quality of grapefruit. Further studies are required for detailed interpretation, because this wavelength range includes information about molecular vibration and electronic transition.

Thus, TOF-NIRS measurement was superior to the conventional NIR technique in predicting the SSC and acidity of grapefruit. As indicated in Table 2, TOF-NIRS is superior to the conventional NIR technique with respect to measurement time. TOF-NIRS measurement could be adapted for online measurement in the future because of its advantages of high prediction accuracy and short measurement time.

Analysis of variability in single-pulsed laser measurements, with a short time domain (TOF-NIRS), was used to estimate SSC and acidity in grapefruit, in which the wavelength was varied to obtain the best prediction model. The best prediction model for SSC and acidity was obtained at $\lambda=770$ and $760 \mathrm{~nm}$, respectively, which relates to absorption as a result of $\mathrm{OH}$ groups. A comparison of TOF-NIRS to the conventional NIR technique showed that TOF-NIRS was superior from the viewpoint of prediction accuracy and measurement time. TOF-NIRS could be adapted for online measurement with the benefit of high prediction accuracy and short measurement time.

Table 2. Partial least squares regression results for soluble solids concentration (SSC) and acidity content in grapefruit by the time-of-flight nearinfrared spectroscopy (TOF-NIRS) versus near-infrared spectroscopy (NIRS).

\begin{tabular}{lccccccc}
\hline & Objective variable & Wavelength $(\mathrm{nm})$ & Rank $^{\mathrm{z}}$ & $r$ & ${\text { RMSECV }(\%)^{\mathrm{y}}}^{\text {RPD }^{\mathrm{x}}}$ Measuring time $^{\mathrm{w}}$ \\
\hline TOF-NIRS & SSC & 770 & 2 & 0.72 & 0.35 & 1.49 & $10 \mathrm{~ns}$ \\
TOF-NIRS & Acidity & 760 & 2 & 0.85 & 0.06 & 2.19 \\
NIRS & SSC & $500-1010$ & 6 & 0.67 & 0.32 & 1.68 \\
NIRS & Acidity & $500-1010$ & 7 & 0.74 & 0.07 & 1.84 & $30 \mathrm{~s}$ \\
\hline
\end{tabular}

${ }^{\mathrm{z}}$ Number of partial least squares components.

${ }^{y}$ Root mean square error of cross-validation.

${ }^{x}$ Ratio of performance to SD.

wThe measuring time consuming per one sample. 


\section{Literature Cited}

Gributs, C.E.W. and D.H. Burns. 2003a. Multiresolution analysis for quantification of optical properties in scattering media using pulsed photon time-of-flight measurements. Anal. Chim. Acta 490:185-195. Gributs, C.E.W. and D.H. Burns. 2003b. Haar transform analysis of photon time-of-flight measurements for quantification of optical properties in scattering media. Appl. Opt. 42:2923-2930.

Kurata, Y., Y. Ikemoto, and S. Tsuchikawa. 2008. Application of timeof-flight near infrared spectroscopy to fruits-Permeability of pulsed laser beam into satsuma mandarin, white grapefruit and Fuji apple. J. Near Infrared Spectrosc. 16:139-142.

Kurata, Y. and S. Tsuchikawa. 2009. Application of time-of-flight near infrared spectroscopy to fruits: Analysis of absorption and scattering conditions of near-infrared radiation using cross-correlation of the time resolved profile. Appl. Spectrosc. 63:306-312.

Leonardi, L. and D.H. Burns. 1997. Quantitative constituent measurements in scattering media from statistical analysis of photon time-offlight distribution. Anal. Chim. Acta 348:543-551.

Leonardi, L. and D.H. Burns. 1999a. Quantitative measurements in scattering media: Photon time-of-flight analysis with analytical descriptors. Appl. Spectrosc. 53:628-636.

Leonardi, L. and D.H. Burns. 1999b. Quantitative multiwavelength constituent measurements using single-wavelength photon time-offlight correction. Appl. Spectrosc. 53:637-646.
Nicolai, B.M., B.E. Verlinden, M. Desmet, S. Saevels, W. Saeys, K. Theron, R. Cubeddu, A. Prifferi, and A. Torricelli. 2008. Time resolved and continuous wave NIR reflectance spectroscopy to predict soluble solids content and firmness of pear. Postharvest Biol. Technol. 47:68-74.

Oki, Y., M. Maeda, and M. Tanaka. 2004. Development of integrated tunable laser system for laser spectroscopy. Mol. Crystals Liquid Crystals 424:55-63.

Pandozzi, F. and D.H. Burns. 2007. Power law analysis estimates of analyte concentration and particle size in highly scattering granular samples from photon time-of-flight measurements. Anal. Chem. 79: 6792-6798.

Tsuchikawa, S. and T. Hamada. 2004. Application of time-of-flight near infrared spectroscopy for detecting sugar and acid contents in apples. J. Agr. Food Chem. 52:2434-2439.

Valero, C., M. Ruiz-Altisent, R. Cubeddu, A. Pifferi, P. Taroni, A. Torricelli, G. Valentini, D. Johnson, and C. Dover. 2004. Selection models for the internal quality of fruit, based on time domain laser reflectance spectroscopy. Biosystems Eng. 88:313323.

Vanoli, M., P.E. Zerbini, L. Spinelli, A. Torricelli, and A. Rizzolo. 2009. Polyuronide content and correlation to optical properties measured by time resolved reflectance spectroscopy in 'Jonagored' apples stored in normal and controlled atmosphere. Food Chem. 115:1450-1457. 